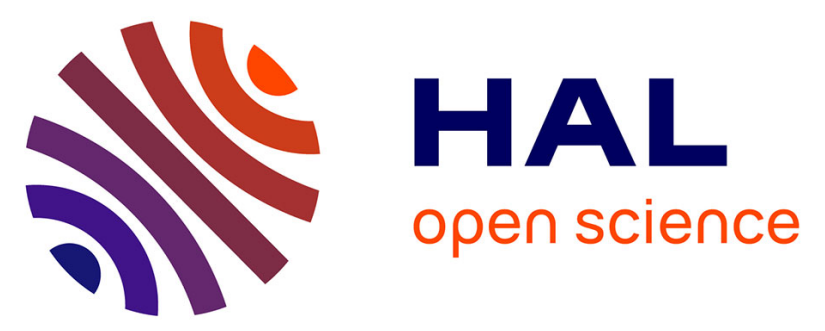

\title{
Lifetime MAP reconstruction in frequency-domain fluorescence lifetime imaging microscopy
}

Philippe Roudot, Charles Kervrann, François Waharte, Jérôme Boulanger

\section{To cite this version:}

Philippe Roudot, Charles Kervrann, François Waharte, Jérôme Boulanger. Lifetime MAP reconstruction in frequency-domain fluorescence lifetime imaging microscopy. IEEE International Conference on Image Processing, ICIP 2012, Oct 2012, Orlando, FL, United States. hal-00763803

\section{HAL Id: hal-00763803 https://hal.inria.fr/hal-00763803}

Submitted on 11 Dec 2012

HAL is a multi-disciplinary open access archive for the deposit and dissemination of scientific research documents, whether they are published or not. The documents may come from teaching and research institutions in France or abroad, or from public or private research centers.
L'archive ouverte pluridisciplinaire HAL, est destinée au dépôt et à la diffusion de documents scientifiques de niveau recherche, publiés ou non, émanant des établissements d'enseignement et de recherche français ou étrangers, des laboratoires publics ou privés. 


\section{LIFETIME MAP RECONSTRUCTION IN FREQUENCY-DOMAIN FLUORESCENCE LIFETIME IMAGING MICROSCOPY}

Philippe Roudot, Charles Kervrann

\author{
Inria Centre de Rennes - Bretagne Atlantique \\ 35042 Rennes Cedex, France
}

\begin{abstract}
We propose a robust statistical framework for reconstructing lifetime map corrupted by vesicle motion in frequency domain FLIM imaging. Instrumental noise is taken into account to improve lifetime estimation. Robust M-estimators and MLestimators allow to jointly estimate motion and lifetime. Performances are demonstrated on simulated and real samples.
\end{abstract}

Index Terms - FLIM, frequency-domain, fluorescence, spot tracking, template matching, M-estimation

\section{INTRODUCTION}

Fluorescence lifetime imaging microscopy (FLIM) is now a widely spread imaging technique for sensing fluorophore environment in a living biological sample (like $\mathrm{pH}$, ions...). Fluorescence lifetime (i.e. average time of a fluorophore staying in excited state before relaxing to its ground state and possibly emitting a photon) is particularly useful to detect the Förster resonance energy transfer (FRET) which can allow to quantify spatial proximity between neighboring molecules [1].

Compared with the well-established time-correlated singlephoton counting (TCSPC) technique, frequency-domain (FD) methods are known to be faster [2]. Nevertheless, in addition to the instrumental noise, vesicles motion may corrupt lifetime results [2]. Thereby we propose a framework that exploits the FD FLIM output intensity model to jointly estimate lifetimes and trajectories of vesicles.

To our knowledge, no previous work have been done on this specific topic; Fourier transform or sine fitting is often recommended to estimate lifetime in the case of static molecules [3, 4]. However vesicle motion estimation is a heavily studied topic and most of methods rely on particle linking after spot detection in each frame [5],[6]. Spot detection can be performed using for example multi-scale wavelet analysis, top-hat filtering or local curvature analysis (see [7]).

In this paper we propose and extension of our work [8] which takes the Intensified Charged Couple Device (ICCD) Poisson-Gaussian noise model into account. The new estimation scheme improves the accuracy of both background and vesicle lifetime estimation.
François Waharte, Jérôme Boulanger

\author{
UMR 144 CNRS - PICT IBiSA - Institut Curie \\ 75248 Paris V, France
}

The remainder of this paper is organized as follows: Section 2 presents the physical process related to FD FLIM measurements. Section 3 describes our image and noise models and presents a procedure devoted to the estimation of lifetime and movement. Section 4 presents experimental results.

\section{FLUORESCENCE LIFETIME MEASUREMENT BY HOMODYNE DETECTION}

FD FLIM images have a very specific intensity footprint which needs to be described for a better understanding of our approach. This section briefly presents the theoretical basis for lifetime measurement in FD-FLIM by homodyne detection (see [4] for a more detailed description).

Given a fluorescence sample excited with a single laser pulse, the impulse response $R(t)$ can be described by a sum of exponential function parameterized by decay times: these lifetimes represent the average time between its excitation time and return time to the ground state of each molecule present in the sample. For the sake of simplicity, we assume the fluorescence decay to be described by a single exponential decay $R(t)=R_{0} e^{-\frac{t}{\tau}}$ where $R_{0}$ is the intensity at $t=0$ and $\tau$ is the decay time.

Whereas the excitation source in time-domain methods is a laser pulse (like with TCPSC), the FD source is a sinusoidally modulated excitation $E(t)$ defined as:

$$
E(t)=C_{E}+A_{E} \sin \left(\omega_{E} t+\Phi_{E}\right)
$$

where $\omega_{E}$ denotes the radial frequency of the excitation signal, $C_{E}$ the offset, $A_{E}$ the amplitude and $\Phi_{E}$ is the phase delay. Hence, the emitted fluorescent signal is defined as :

$$
\begin{aligned}
F(t) & =E(t) * R(t) \\
& =C_{F}+A_{F} \sin \left(\omega_{E} t+\Phi_{E}-\arctan \left(\omega_{E} \tau\right)\right)
\end{aligned}
$$

where $*$ denotes the convolution operator. Accordingly, the phase delay $\Phi_{E}-\arctan \left(\omega_{E} \tau\right)$ of $F(t)$ allows us to recover $\tau$. In an experimental setup, the frequency $(>1 \mathrm{MHz})$ is too high to be compatible with CCD sensor frame rate. Therefore the signal $F(t)$ is phase-modulated with $K$ sinusoidal signals 

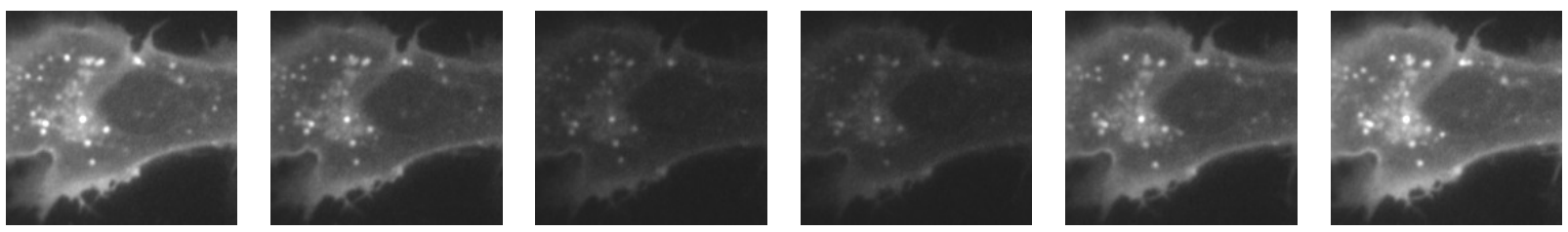

Fig. 1. An example of FD FLIM measurement with $K=6$ phase-modulation signal.
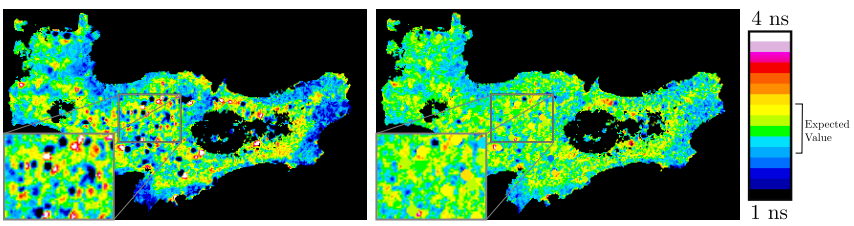

Fig. 2. Left: Lifetime map using Fourier decomposition. Dark blue and red spots are irrealistic values due to organel movements. Right: lifetime map reconstruction using our method.

$M_{k}(t), k \in[1, K]$ at frequency $\omega_{E}$ (homodyne detection):

$$
M_{k}(t)=C_{M}+A_{M} \sin \left(\omega_{E} t+\Phi_{M_{0}}+\frac{2 \pi k}{K}\right) .
$$

Thanks to the low pass effect of the CCD detector, the higher frequencies of the phase modulated-signal $F(t) M_{k}(t)$ are negligible. Therefore we obtain $K$ time-independent signals described as a function of $k \in[1, K]$ (see Fig. 1):

$$
S_{\theta}(k)=\mathcal{G}_{\mathrm{CCD}}\left(F(t) M_{k}(t)\right)=C_{S}+A_{S} \cos \left(\frac{2 \pi k}{K}+\Phi_{\tau}\right)
$$

where $C_{S}=C_{F} C_{M}, A_{S}=A_{F} A_{M}$ and $\Phi_{\tau}$ is the phase. In what follows, we denote $\theta=\left(C_{S}, A_{S}, \Phi_{\tau}\right)^{T}$. The phase $\Phi_{\tau}=\left(\Phi_{M_{0}}-\Phi_{E}+\arctan \left(\omega_{E} \tau\right)\right)$ can be recovered using sine fitting or Fourier decomposition techniques from the lower frequency of $S_{\theta}(k)$. The phase delay $\left(\Phi_{M_{0}}-\Phi_{E}\right)$ of the system is calibrated by recording a reference frame sequence of a fluorescent sample with known lifetime. Finally, as we monitor the frequency $\omega_{E}, \tau$ comes straightforwardly. Figure 2 shows an example of lifetime computation using the Fourier transform and specific locations highlights the problem of moving vesicles which generate unrealistic values.

\section{LIFETIME MAP RECONSTRUCTION METHOD}

The method presented above has been successfully applied on immobilized samples [2]. However we can expect to improve lifetime estimation by taking instrumental noise into account, furthermore considering moving vesicles is crucial when studying lifetime accuracy. In this section, we propose a statistical framework providing a more precise reconstruction of the lifetime map. First we present the image and noise modeling, secondly we describe the lifetime estimator procedure of both background and subresolution vesicles.

\subsection{Modeling spatial and temporal variations}

Several degradations were taken into account to model the vesicle intensity. A Gaussian function approximates the point spread function (PSF) which corresponds to a Bessel function in theory. As the confocal equipment is not rigorously stigmatic the signal is composed of a background and a pinpoint vesicle before microscope diffraction. The background follows the model (4) due to cytoplasmic fluorescence. Finally the background is assumed to be smooth over the PSF support. More formally, let $I(k)(x)$ be the intensity on frame $k$ at location $x \in \Omega$ (pixel grid):

$$
I(k)(x)=S_{\theta_{b}}(k)(x)+S_{\theta_{0}}(k) G_{\gamma}\left(x-x_{0}(k)\right)+\varepsilon(k)(x)
$$

where $S_{\theta_{0}}(k)$ is the vesicle mean intensity parameterized by $\theta_{0}=\left(C_{S_{0}}, A_{S_{0}}, \Phi_{\tau_{0}}\right)^{T}$ as in (4), $S_{\theta_{b}}(k)(x)$ is the spatially varying background signal parameterized by $\theta_{b}(x)=$ $\left(C_{S_{b}}(x), A_{S_{b}}(x), \Phi_{\tau_{b}}(x)\right)^{T}, G_{\gamma}(x)$ is a Gaussian function of variance $\gamma^{2}$ describing the shape of the vesicles and centered on the origin, $x_{0}(k)$ is the vesicle location on frame $k, \varepsilon$ is the noise introduced by the ICCD and described in the next section.

\subsection{Noise modeling}

In this section we characterize the noise induced by the ICCD setup. We present an alternative approach to the derivation described in [3].

In our setup, ICCD is composed of a CCD sensor and a third generation image intensifier which amplifies the photonic signal of the sample. In a nutshell, intensifiers photocathode transforms photons into electrons which are concentrated and accelerated in microchannel plates, the ouput electrons are transformed back into burst of photons using a phosphor screen. As a result, the spatially correlated photons output follows a filtered Poisson process. Considering CCD gain and read out noise, Boddeke showed that the ICCD response $I(x)$ at location $x$ in $\Omega$ is [9]:

$$
I(x)=g_{\mathrm{INT}} g_{\mathrm{cCD}} \aleph(x)+\xi
$$

where $\aleph(x)$ is the incident photon number on the ICCD which follows a Poisson law of parameter $\lambda(x), \xi$ is the CCD read out Gaussian noise such as $\xi \sim \mathcal{N}\left(m_{\xi}, \sigma_{\xi}^{2}\right), g_{\text {ccD }}$ is the gain of the CCD sensor and $g_{\mathrm{INT}}$ is the gain of the intensifier assumed to be a realization of a random variable. Indeed if CCD gain is often considered as constant, Boddeke experimentally showed that phosphor screen uncontrolled emission impact is non-negligible so that the resulting gain cannot be considered as constant [9]. Under the proper assumptions of 


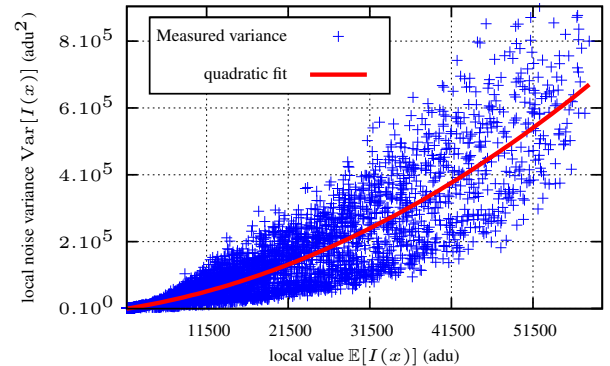

Fig. 3. Robust fit between $\mathbb{E}[I(k)(x)]$ and $\operatorname{Var}[I(k)(x)]$ using a phase calibration stack.

independence of random variables it comes:

$$
\begin{aligned}
\operatorname{Var}[I(x)]= & g_{\mathrm{CCD}}^{2} \operatorname{Var}\left[g_{\mathrm{INT}} \aleph(x)\right]+\sigma_{\xi}^{2} \\
= & g_{\mathrm{CCD}}^{2} \operatorname{Var}\left[g_{\mathrm{INT}}\right] \operatorname{Var}^{2}[\aleph(x)] \\
& +g_{\mathrm{CCD}}^{2}\left(\mathbb{E}^{2}\left[g_{\mathrm{INT}}\right]+\operatorname{Var}\left[g_{\mathrm{INT}}\right]\right) \operatorname{Var}[\aleph(x)]+\sigma_{\xi}^{2}
\end{aligned}
$$

By taking the expectation of (6) and knowing that $\operatorname{Var}[\aleph(x)]=$ $\lambda(x)=\mathbb{E}[\aleph(x)]$, we have :

$$
\begin{aligned}
\operatorname{Var}[I(x)]= & \frac{\operatorname{Var}\left[g_{\mathrm{INT}}\right]}{\mathbb{E}^{2}\left[g_{\mathrm{INT}}\right]}\left(\mathbb{E}[I(x)]-m_{\xi}\right)^{2} \\
& +g_{\mathrm{CCD}}\left(\mathbb{E}\left[g_{\mathrm{INT}}\right]+\frac{\operatorname{Var}\left[g_{\mathrm{INT}}\right]}{\mathbb{E}\left[g_{\mathrm{INT}}\right]}\right)\left(\mathbb{E}[I(x)]-m_{\xi}\right)+\sigma_{\xi}^{2} .
\end{aligned}
$$

As proposed in [10] where a linear noise model is considered on CCD cameras, we estimate the parameters of the quadratic relation using a robust M-estimator. Each image of the stack is first divided in $7 \times 7$ tiles. The values $\mathbb{E}[I(x)]$ are approximated by the median of the tile pixels and $\operatorname{Var}[I(x)]$ values are approximated using least trimmed square estimation of the pseudo-residual variance. Figure 3 shows an example of robust fitting on a phase calibration stack. In what follows, $\operatorname{Var}[I(x)]$ is estimated using the parameter ruling the quadratic relation (8) and the average local image value for $\mathbb{E}[I(x)]$.

\subsection{Background lifetime estimation}

Due to the high number of photon on the ICCD output we can consider the Poisson-Gaussian noise as Gaussian noise with a spatially varying variance $\operatorname{Var}[I(k)(x)]$. This model enables to cope with non-stationary noise and select appropriately the level of confidence. Thus, the background lifetime parameters are estimated by minimizing the following least mean square criterion:

$$
\hat{\theta}_{b}=\underset{\theta_{b}}{\operatorname{argmin}} \sum_{k \in[1, K]} \frac{\left(I(k)(x)-S_{\theta_{b}}(k)(x)\right)^{2}}{\operatorname{Var}[I(k)(x)]}
$$

where $\operatorname{Var}[I(k)(x)]$ is computed by applying the relation (7) on pixel value $I(k)(x)$. Figure 4 shows the enhancement provided by taking into account heteroscedasticity of the noise.

\subsection{Vesicle parameter estimation}

This section presents the estimation procedure of the decay time $\tau_{0}$ for each detected vesicles. In this procedure, the vesicle location $x_{0}(k)$ and shape $\gamma$ and the lifetime parameter $\theta_{0}$ are jointly estimated. This optimization problem is implemented by successive minimization of criteria with respect to $\left(x_{0}(k), \gamma\right)$ and to $\theta_{0}$ until $\left|\frac{\theta_{0}^{n}-\theta_{0}^{n+1}}{\theta_{0}^{n}}\right| \leq \delta$ with $n$ the iteration number and $\delta$ a constant (set to $10^{-6}$ in our experiments).

\subsubsection{Iterative minimization procedure}

The lifetime parameters $\theta_{0}$ are estimated by minimizing a weighted least square similar to (9). At iteration $n$ of the minimization algorithm we have :

$$
\hat{\theta}_{0}^{n}=\underset{\theta_{0}}{\operatorname{argmin}}
$$

$$
\sum_{k \in[1, K]} \sum_{x \in W\left(\hat{x}_{0}^{n}(k)\right)} \frac{\left(I(k)(x)-S_{\theta_{0}}(k) G_{\gamma^{n}}\left(x-\hat{x}_{0}^{n}(k)\right)\right)^{2}}{\operatorname{Var}[I(k)(x)]}
$$

where $W\left(x_{0}^{n}(k)\right)$ is a $7 \times 7$ window centered at $\hat{x}_{0}^{n}(k)$.

The motion parameters $x_{0}^{n+1}(k)$ are estimated for each frame $I(k), k \in[1, K]$ given $\hat{\theta}_{0}^{n}$. Tracking is performed frame-by-frame by minimizing a robust error function, at iteration $n+1$ of the algorithm we have :

$$
\begin{aligned}
& \left(\hat{x}_{0}^{n+1}(k), \hat{\gamma}^{n+1}\right)=\underset{\left(x_{0}(k), \gamma\right)}{\operatorname{argmin}} \\
& \sum_{x \in W\left(x_{0}(k)\right)} \rho_{\eta}\left(I(k)(x)-S_{\hat{\theta}_{0}^{n}}(k) G_{\gamma}\left(x-x_{0}(k)\right)\right) .
\end{aligned}
$$

The function $\rho$ is an influence function chosen and $\eta=$ $\lambda \sigma_{r e s}(k)$ is a scale parameter used to to weight down outliers during the minimization process. Experiments show that the Leclerc influence function defined as $\rho_{\eta}(r)=1-e^{-\frac{r^{2}}{\eta^{2}}}$ gives more stable results even if no significant difference is noticeable with other M-estimators (e.g. Tukey Bi-weighted or German-McClure). The scale parameter $\sigma_{r e s}(k)$ is defined for each frame as [11]:

$$
\sigma_{\text {res }}(k)=1.4826 \operatorname{median}_{i \in\left[1,\left|W\left(\hat{x}_{0}(k)\right)\right|\right]}\left|r_{i}-\operatorname{median}_{i \in\left[1,\left|W\left(\hat{x}_{0}(k)\right)\right|\right]} r_{i}\right|
$$

where $\left\{r_{i}\right\}_{i \in\left[1, \mid W\left(x_{0}(k) \mid\right]\right.}$ is the set of residuals of estimation introduced in (11) when using the $L^{2}$-norm as influence function which is equivalent to a least mean square estimation.

\subsection{2. initialization}

Motion $x_{0}^{0}(0)$ are initialized by over detecting spots using derivative of Gaussian filtering. Then Gaussian fitting [12] is applied to initialize $x_{0}^{0}(k)$ and $\gamma^{0}$. Then, for each iteration $n$, we set : $\gamma^{n+1}=\hat{\gamma}^{n}, x_{0}^{n+1}(k+1)=\hat{x}_{0}^{n+1}(k-1)$, for $k \in[1, K-1]$ and $x_{0}^{n+1}(0)=\hat{x}_{0}^{n}(0)$. At each iteration, 

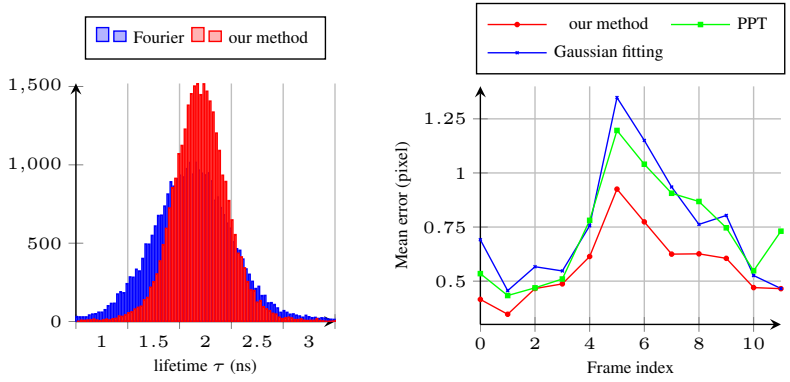

(Section 3.3), then by drawing a patch of size $\hat{\gamma}$ and value $\hat{\tau}_{0}$ at location $\hat{x}_{0}(0)$ for each vesicle on the background lifetime map.

\section{CONCLUSION}

Our method allows to reconstruct lifetime map of sample usually corrupted by vesicle motion in a efficient and fast way in FD FLIM imaging. This study shows the importance of taking the instrumental noise and the intensity model into account while tracking vesicles. Other trackers such as linking method will be compared to assess the differences in performance of our procedure which is much less demanding in computation compared to spot detection at each frame.

\section{ACKNOWLEDGEMENT}

Table 1. Convergence of tracking and lifetime errors is reach after 3 iteration on 30 simulated vesicles and $95 \%$ of vesicle tracked.

lifetime parameters $\theta_{0}^{n}=\left(C_{S_{0}}^{n}, A_{S_{0}}^{n}, \Phi_{\tau_{0}}^{n}\right)^{T}$ are initialized as follows : $C_{S_{0}}^{n}$ and $A_{S_{0}}^{n}$ are respectively the mean and the maximum value of the set $\left\{\frac{I(k)(x)}{G_{\gamma^{n}}\left(x-x_{0}^{n}(k)\right)}\right\}_{k \in[1, K], x \in W\left(x_{0}^{n}\right)(k)}$. $\Phi_{\tau_{0}}^{n}=\Phi_{M_{0}}-\Phi_{E}+\arctan \left(\omega_{E} \tau_{E G F P}\right)$ where $\tau_{E G F P}$ is the fluophore lifetime measured without FRET (e.g. $2.5 \mathrm{~ns}$ for the EGFP) and $\left(\Phi_{M_{0}}-\Phi_{E}\right)$ is obtained by calibration.

\section{RESULTS AND DISCUSSION}

This section presents our experimental results conducted on both synthetic and real samples.

Synthetic data Our simulations are based on our intensity model presented in Section 3.1 and the noise model presented in Section 3.2. Background is simulated with an experimental vesicle-free sample and cytoplasmic fluorescence is simulated in the background with constant parameter $\theta_{b}$. Vesicle intensities follow (5) and motions are limited to a two a displacement of pixels. The non-stationary Gaussian noise parameters are estimated on the stack and then imposed on synthetic vesicles. Table 1 shows the convergence of lifetime and motion parameter on simulated vesicles.

Real samples Experiments were conducted on living cells using a confocal microscope with spinning disk setup (UMR 144 CNRS-PICT Institut Curie). The intensifier is a third generation from Lambert Instruments (model II18MD). Figure 4 exhibits the accuracy of our lifetime estimation method presented Section 3.3 on a real sample, showing an improvement of the standard deviation from 0.48 to 0.27 . This enhancement is consistent with expected results by biologist experts. Figure 4 also highlights our tracking results (70 \% of completed track) on the same cell with 30 moving vesicles we previously tracked manually using the MJtrack software [7]. We compare our tracker with Gaussian fitting and the particle tracker plugin in ICY and presented in [13] after normalization. Figure 2 shows the reconstruction of the lifetime map. It is built by estimating lifetime on the background

The authors thanks Cedric Blouin for providing the experimental data.

\section{REFERENCES}

[1] M.A. Hink, T. Bisseling, and A.J.W.G. Visser, "Imaging proteinprotein interactions in living cells," Plant molecular biology, vol. 50, no. 6, pp. 871-883, 2002.

[2] K. Suhling, P.M.W. French, and D. Phillips, "Time-resolved fluorescence microscopy," Photochemical \& Photobiological Sciences, vol. 4, no. 1, pp. 13-22, 2005.

[3] BQ Spring and RM Clegg, "Image analysis for denoising fullfield frequency-domain fluorescence lifetime images," Journal of Microscopy, vol. 235, no. 2, pp. 221-237, 2009.

[4] J.R. Lakowicz and B.R. Masters, "Principles of fluorescence spectroscopy," Journal of Biomedical Optics, vol. 13, pp. 029901, 2008.

[5] E. Meijering, I. Smal, and G. Danuser, "Tracking in molecular bioimaging," IEEE Signal Processing Magazine, vol. 23, no. 3, pp. 46-53, 2006.

[6] N. Chenouard, A. Dufour, and J.C. Olivo-Marin, "Tracking algorithms chase down pathogens," Biotechnology Journal, vol. 4, no. 6, pp. 838845, 2009.

[7] I. Smal, M. Loog, W. Niessen, and E. Meijering, "Quantitative comparison of spot detection methods in fluorescence microscopy," IEEE Transactions on Medical Imaging, vol. 29, no. 2, pp. 282-301, 2010.

[8] P. Roudot, C. Kervrann, and F. Waharte, "Lifetime estimation of moving vesicles in frequency-domain fluorescence lifetime imaging microscopy," in IEEE International Symposium on Biomedical Imaging: Nano to Macro, 2012., 2012, pp. 668-671.

[9] F.R. Boddeke, Quantitative fluorescence microscopy, Ph.D. thesis, Delft University Press, 1999.

[10] J. Boulanger, C. Kervrann, P. Bouthemy, P. Elbau, JB Sibarita, and J. Salamero, "Patch-based non-local functional for denoising fluorescence microscopy image sequences," IEEE Transactions on Medical Imaging, vol. 28, pp. 12, 2009.

[11] P.J. Rousseeuw, A.M. Leroy, and J. Wiley, Robust regression and outlier detection, vol. 3, Wiley Online Library, 1987.

[12] C.M. Anderson, G.N. Georgiou, IE Morrison, GV Stevenson, and R.J. Cherry, "Tracking of cell surface receptors by fluorescence digital imaging microscopy using a charge-coupled device camera.," Journal of Cell Science, vol. 101, no. 2, pp. 415, 1992.

[13] A. Genovesio, T. Liedl, V. Emiliani, W.J. Parak, M. Coppey-Moisan, and J.C. Olivo-Marin, "Multiple particle tracking in 3-d+t microscopy: Method and application to the tracking of endocytosed quantum dots," IEEE Transactions on Image Processing, vol. 15, no. 5, pp. 1062-1070, 2006. 